\title{
Pre-anesthesia Check of Breathing Gireulit - Is it infallible?
}

Madhu Gupta, Shalini Subramaniam, Deepika Govil, Iti Shri

From the Department of Anaesthesia, ESI Post Graduate Institute of Medical

Sciences \& Research, Basaidarapur, Ring Road, New Delhi 110015 , India.

\begin{abstract}
:
It is a case of equipment failure in the form of leak in the breathing circuit which remained undetected during the pre-anaesthesia leak test done before the start of two different cases. The peri-operative course of the first case was uneventful. The second patient developed intra-operative bronchospasm. But suddenly the ventilator bellows collapsed and manual ventilation was possible only with intermittent oxygen flushes. Presence of a hissing sound prompted a check of the breathing system and revealed a hole $2.5 \mathrm{~mm}$ size in the expiratory limb of the silicone breathing hose. It probably got resulted when the circuit was stretched while trying to ventilate against an increased airway resistance and could be found. We recommend extending our breathing circuit to its the full length while checking to avoid such misses as a standard practice.
\end{abstract}

Key words: Anesthesia, Bronchial Spasm, Ventilators, Equipment Failure, Oxygen.

\section{Introduction}

Checking anesthesia equipment properly prevents equipment related morbidity and mortality, improves preventive maintenance and educates the anesthesia provider about equipment. Failure to do a proper anesthesia setup results in patient injury and near misses.

Breathing circuit leak, obstruction or misconnects contribute to around $20 \%$ of the critical incidents occurring during anesthesia which if not immediately identified may result in serious harm [1]. As anesthesia machines becoming more electronic, more checking is performed automatically and it is for the anesthesia personnel to remain aware of which anesthesia components are tested by these automated self tests and which ones are not [2]. Faults may develop during anesthesia's which were either not present or not apparent on the preoperative equipment check. We here present a case of detection of a leak from breathing circuit which emphasize on the above fact as well as that an alert anesthesiologist may avert an adverse anesthetic outcome.

\section{Case Report}

The elective cases in the surgery operating room (OR) were started after routine automated preuse check of the anesthesia machine (Blease Sirius, Spacelabs Healthcare Ltd., USA) and

Corresponding Author: Dr. Madhu Gupta

Email: madhugupta2602@gmail.com

Received: November 30, 2013 | Accepted: December 24, 2013 | Published Online: December 30, 2013 This is an Open Access article distributed under the terms of the Creative Commons Attribution License (creativecommons.org/licenses/by/3.0)

Conflict of interest: None declared | Source of funding: Nil | DOl: http://dx.doi.org/10.17659/01.2013.0108 
equipment including the breathing circuit to be used (as specified by the manufacturer as well as recommended by the FDA 2008 checklist). The first patient was a 43 year old woman posted for breast lump excision. After routine intravenous induction, she was intubated and put on volume controlled ventilation. The case went on uneventfully and she was reversed and extubated without a consequence. The second patient, a 37-year-old female, posted for open cholecystectomy, had a history of allergic asthma and allergy to several agents including milk, egg, soya and ampicillin. She was on salbutamol and budecort puffs twice a day. Preoperatively, bilateral vesicular breath sounds with no adventitious sound were auscultated. After premedication with hydrocortisone $100 \mathrm{mg}$, midazolam $1 \mathrm{mg}$ and fentanyl $2 \mu \mathrm{g} / \mathrm{kg}$ intravenously, anaesthesia was induced with thiopentone $225 \mathrm{mg}$, vecuronium $5 \mathrm{mg}$ and sevoflurane and intubated. Bilateral air entry was confirmed, with no adventitious sounds and put on volume control ventilation. A minute later suddenly her peak airway pressure (Paw) increased to $35 \mathrm{~cm} \mathrm{H}_{2} \mathrm{O}$. Bilateral wheeze was present on auscultation. The saturation was $100 \%$, end tidal $\mathrm{CO}_{2}$ was $40 \mathrm{mmHg}$ and the capnogram showed an upward sloping. But paradoxically the ventilator bellows were collapsing. She was taken immediately on manual ventilation, with APL valve fully closed, intermittent oxygen flushes were given. Still, the reservoir bag did not fill effectively. Simultaneously, the presence of a hissing noise alerted us to the presence of a leak somewhere. A quick and systematic survey of the breathing system revealed a leak from the silicone rubber breathing circuit, close to the point of attachment at the machine end. Immediately the patient was taken on Bain's circuit to facilitate ventilation. The bronchospasm was managed pharmacologically with salbutamol and ipravent puffs, increasing the inhaled sevoflurane concentration. The patient was sans wheeze and the Paw decreased to $18 \mathrm{~cm} \mathrm{H}_{2} \mathrm{O}$. The rest of the case was completed uneventfully using a new disposable breathing circuit. The patient was reversed and extubated uneventfully. Later, on examining the defective breathing tube, a rent $2.5 \mathrm{~mm}$ in diameter was identified in the expiratory limb of the breathing circuit [Fig.1].

\section{Discussion}

Breathing circuit misconnects and disconnects are one of the most common cause of anesthetic injury [3]. Our institute has the protocol of daily pre-anesthesia checkout of the anesthesia machine based on the 2008 recommendations and the manufacturer recommended automatic self check [4]. The same had been done along prior to the start of the first case. During the electronic checkout, the compliance of the circuit was measured and the compensation volume of the ventilator calculated. This procedure also looks for leaks in the ventilator, $\mathrm{CO}_{2}$ canister, unidirectional valves, breathing circuit, $Y$ piece and APL valve. During the self test, all gas flows should be at zero. The APL valve should be closed and the patient port occluded. The breathing system should be pressurized to $30 \mathrm{~cm} \mathrm{H}_{2} \mathrm{O}$ by using the flush. If there is no leak, the pressure will remain near this level for at least 10 seconds. The APL valve is then opened. The pressure should decrease. The leak can

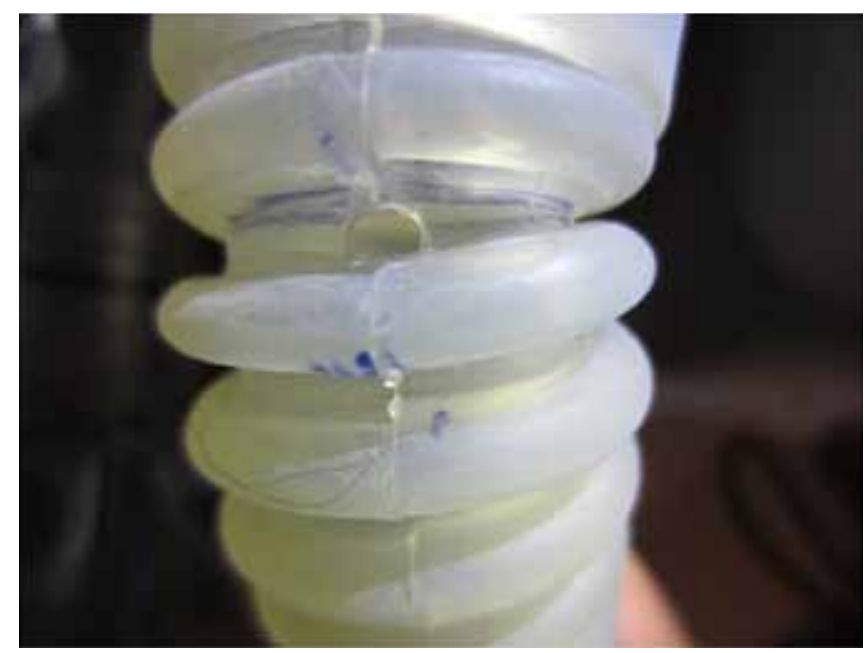

Fig.1: Rent in the circuit that got revealed on extending the corrugations. 
be quantified by adjusting the oxygen flowmeter to maintain a pressure of $30 \mathrm{~cm} \mathrm{H}_{2} \mathrm{O}$ in the breathing system with the $Y$ piece occluded and the APL valve closed. The breathing system standard requires that this does not exceed $300 \mathrm{ml} /$ minute [5]. This was passed successfully.

Before the start of the second case on the same anesthesia machine and the same breathing circuit as per the institutional protocol based on the guidelines, the breathing circuit was subjected to peruse test as mentioned above and it passed the test. Induction during second case was uneventful until the patient developed bronchospasm due to her preexisting allergic disorder and ventilation against an increased airway resistance was attempted. The leak was revealed at this juncture. This could have happened while trying desperate attempts to ventilate the patient and the breathing circuit was stretched to the extent that the hole got manifested as hissing noise and hence a leak. Had it not been the alertness of the anaesthesiologist in recognizing the hiss, this could be a serious event and the reason gone undetected.

We tried to assess whether the leak was present even before the first case or developed in between the cases, and if it was present from the beginning why it did not reveal earlier. It was unlikely that any damage occurred to the tube between the two cases as there was no mishandling or insult to the tube, like getting crushed under the wheels of the anaesthesia machine. Secondly, the edges of the puncture appeared smooth and long standing and not ragged and fresh; indicating that the rent was present even before the start of first case. The injury to the tube seemed to have been inflicted by a sharp object; either a stab from a blade or uncapped needles on the anaesthesia machine.

The manufacturer's recommendations regarding cleaning and sterilization of breathing circuits had been meticulously followed. They are washed with soap and detergent by immersing them vertically in this solution so that the inside of the circuit also makes contact with the cleaning agent, dried completely by hanging them vertically and then autoclaved at $121^{\circ} \mathrm{C}$ for 20 minutes. May be there was some mishandling on the previous day when it was taken for sterilization by the technical staff after the completion of OT list.

The tear was also not evident on casual inspection of the tube but only on stretching the circuit as the tear was present in between the corrugations. The ground facing location of the puncture and the hanging down position of the circuit were responsible for close approximation of the edges of the opening. This had enabled the leak test to be passed both the times. Possibly during the whole period of anesthesia for first case and the beginning of the second case the hole was sealed in between the corrugations. Later on when the pre-use check was repeated with the defect in other positions like at the patient end or facing superiorly, the leak was at once detected as now the corrugations were brought apart. Stretching or lifting of the circuit during mechanical ventilation in the second patient had caused the leak to be evident as this had brought corrugations apart [6]. The learning from the above episode is that any incident which howsoever may seem to be a trivial one should encourage an accident investigation, reasoning, rectification and formulation of policies for prevention of any such untoward event. First of all, as mentioned the surface of the anaesthesia workstation should not be used to store objects as blade, needles, syringes which might be a detriment for the anesthesia equipment. The technical staff should be sensitised to the significance of careful handling during disinfection, sterilisation, storage and usage of anaesthetic equipment to avoid physical or caustic injury to these valuable equipment. We also suggest that not only the breathing circuit be inspected to determine for damaged or missing parts, presence of foreign bodies [7]; we should routinely extend the tubing to 
its full length prior to the checkout so that the leak within the corrugations can be picked [6].

Equipment malfunction despite careful maintenance and routine pre use check have been reported in almost every part of the anesthesia machine including sampling port [8], ventilator system [9].

This incident highlights that standard maintenance; complete pre-use check and previous uneventful use do not give surety against intra-operative machine error. It stresses the importance of eternal vigilance, speedy and methodical assessment of breathing system disconnects and the availability of alternate $\mathrm{O}_{2}$ source and manual resuscitator so that the primary event does not progress to the point of patient injury. We also stress that the preanaesthesia check guidelines for checking leak in the breathing circuit to be further specific in emphasising the need for stretching the breathing circuit to be used to be stretched to the full length while testing so that the leak within the corrugations can be picked $[10,11]$. The present guidelines are silent on this [4].

\section{References}

1. Cooper JB, Newbower RS, Long CD, McPeek BJ.Preventable anesthesia mishaps: A study of human mishaps. Anesthesiology. 1978;49:399406.

2. Miller RD. Miller's Anesthesia. 7th Edition. Philadelphia: Elsevier Churchill Livingstone
2010:669.

3. Caplan RA, Vistica MF, Posner KL, Cheney FW. Adverse Anesthetic Outcomes Arising from Gas Delivery Equipment A Closed Claims Analysis. Anesthesiology. 1997;84:741-748.

4. Recommendations for Pre-Anesthesia Checkout Procedures (2008). Available from URL https://www.asahq.org/clinical/fda.htm. Accessed June 20, 2013.

5. Chung DC, Ho AM, Tay B. Apnea-volume" warning during normal ventilation of the lungs: an unusual leak in the Narkomed 4 Anesthesia System. Journal of Clinical Anaesthesia. 2001; 13(1):40-43.

6. Dorsch JE, Dorsch SA. Understanding Anesthesia Equipment. $5^{\text {th }}$ edition. Baltimore: Lippincott Williams and Wilkins;2008.934.

7. Dorsch JE, Dorsch SA. Understanding Anesthesia Equipment $4^{\text {th }}$ edition. Baltimore: Lippincott Williams and Wilkins; 2008. 949.

8. Needleman S, Kaplan RF. Unusual Source of Air Leak in a Pediatric Anesthesia Breathing Circuit. AnesthAnalg. 1995;81:654.

9. Rajinikanth $R$, Sundara Rajan. Scavenging: Another Hidden Danger. Out of the blue E-letters. BJA May 2008.

10. Dorsc JE, Dorsch SA. Understanding Anesthesia Equipment. 5th edition. Baltimore: Lippincott Williams and Wilkins; 2008. 942.

11. Neema C. Anesthetic Concerns in Patients with Retinopathy of Prematurity. Journal of Case Reports. 2013;3(1):59-63. 